\title{
La acción de sujetos complejos hacia un Nuevo Humanismo en contextos de crisis civilizatoria*
}

\author{
María Teresa Pozzoli ${ }^{1}$ \\ (D) $h t t p: / / o r c i d . o r g / 0000-0001-7169-9283$ \\ Instituto de pensamiento complejo, Santiago de Chile
}

DOI: http://dx.doi.org/10.17081/eduhum.19.33.2652

Recibido: 3 de noviembre de 2016

Aceptado: 25 de marzo de 2017

\section{Complex actions of subjects towards a new humanism in the contexts of a civilizing crisis}

\author{
Palabras clave: \\ Nuevo Humanismo, \\ Crisis civilizatoria, \\ Cambio actitudinal, \\ Sujeto complejo, \\ Conciencia planetaria.
}

Key words:

New Humanism,

Civilization crisis,

Attitudinal change,

Subject complex,

Planetary consciousness.

\begin{abstract}
Resumen
La humanidad enfrenta una crisis terminal del patrón civilizatorio de la modernidad. Desde el Pensamiento Complejo -en su reemplazo- se promueve la emergencia de un Nuevo Humanismo, una manera diferente de estar en el mundo desde la expresión de una conciencia planetaria. El logro de esta transformación depende de qué tanto estén dispuestos los sujetos complejos a asumir un cambio actitudinal, orientando su acción en consistencia/congruencia con las ideas que enuncian dicho cambio. Este trabajo revela algunas contradicciones del proceso de transformación en curso y propone el uso de algunos recursos de orden conceptual y metodológico que lo facilitan.
\end{abstract}

\begin{abstract}
Humanity faces a terminal crisis of the civilizing pattern of Modernity. From the Complex Thought - in its replacement - the emergence of a New Humanism is promoted - a different way of being in the world from the expression of a planetary consciousness. The achievement of this transformation will depend on the decision of complex subjects to assume the attitudinal change orienting its action in consistency / congruence with the ideas that enunciate the aforesaid change. This paper highlights some contradictions about the ongoing transformation process and proposes the use of some conceptual and methodological resources that facilitate this process.
\end{abstract}

\section{(9) $(\Theta \Theta$}

Referencia de este artículo (APA): Pozzoli, M. (2017). La acción de sujetos complejos hacia un Nuevo Humanismo en contextos de crisis civilizatoria. En Revista Educación y Humanismo, 19(33), 398-408. http://dx.doi.org/10.17081/eduhum.19.33.2652

Artículo de reflexión sobre la acción sujetos complejos.

1. Instituto de pensamiento complejo.mariateresa.pozzoli@gmail.com 


\section{Introducción}

En la última década, ha sido notoria en las ciencias sociales la preocupación paradigmática, su relación con los sujetos, sus modos de ser y vivir, de estar en el mundo.

En tal sentido, es claro que la construcción y organización del conocimiento juega un papel fundamental en los procesos de estabilización o transformación de los sujetos; a su vez, esta construcción tiene un impacto ineludible en lo social, y en esta relación interactiva del Unitas Multiplex se hace evidente el principio hologramático del Pensamiento Complejo (Morin, 1999).

Ghiso (2002) sostuvo que la relación entre conocimiento, la construcción del ser del sujeto y la congruencia de su acción, ha de ser entendida como una inquietud y un desafío que se inscribe en los ámbitos de la responsabilidad y de la eticidad de intelectuales y académicos.

Por otra parte, somos al mismo tiempo testigos y partícipes de los problemas globales que atraviesa la humanidad, y, en consecuencia, objetos y sujetos del patrón civilizatorio en crisis (Boff, 2001; Leff, 2007).

En este contexto civilizatorio crítico, ni la clase política ni la economía ni el conocimiento, la información o la geopolítica han sabido dar respuestas correctivas sino que más bien acentúan los rasgos de una sociedad de riesgo, de peligro constante (Luján \& Echeverría, 2004).

Los modelos disciplinarios en curso parecieran inhabilitados y al mismo tiempo inhiben a los sujetos para desarrollar procesos de reflexividad de segundo orden, que puedan dar cuenta de los supuestos desde los cuales pensamos y actuamos, así como en nuestras posibilidades de ser, hacer, tener, sentir y pensar (Ghiso, 2002). Como afirma Morin (1999): "Las ciencias no tienen conciencia de los principios ocultos que gobiernan sus elucidaciones. Las ciencias no tienen conciencia de que les falta conciencia”. (p.84)

Desde el punto de vista de la subjetividad, convivimos con una sensación pesimista respecto al futuro y vivimos una suerte de reacción adaptativa inercial frente al fracaso (Mora, 2007). No obstante, sabemos que las situaciones de crisis entendidas como oportunidad pueden abrirse al cambio y a la innovación creativa (Riechmann, 2006). Como ya se ha dicho, en este contexto crítico, es necesario revisar nuestras percepciones, transformar el pensamiento $\mathrm{y}$ concentrarnos en la acción educativa transformadora para difundir y acompañar la conformación de un nuevo patrón civilizatorio, basado en los valores de un Nuevo Humanismo cuyo modelo de convivencia ha de ser conformado por ciudadanos globales, es decir, portadores de una conciencia planetaria.

En este trabajo revisamos parte de las contradicciones y obstáculos que enfrenta el sujeto complejo para asumir la decisión de participar con su acción del Nuevo Humanismo, orientando el cambio actitudinal como resultado de 
su desarrollo sociopersonal, necesario en este proceso transformativo*.

\section{Desarrollo}

\section{El contexto de la crisis del patrón civiliza-} torio

De la mano de la crisis del patrón civilizatorio de la Modernidad, asistimos al agotamiento de un modelo de sociedad que se mantiene vigente y que pone en evidencia numerosas contradicciones valóricas en el Unitas Multiplex. Leonardo Boff (2001) afirma que en este contexto "...urge una revolución ética mundial" (p.17). Y realizar esta revolución ética requiere de la educación transformadora del universo actitudinal de los sujetos, que facilitará un pacto ético de ciudadanos globales, respecto del cual nadie quede al margen. Todos los ciudadanos del mundo son alcanzados por esta crisis, $y$, directa o indirectamente, han de ser parte de las soluciones. Diversos autores han propuesto numerosos rótulos para caracterizar ciertos aspectos del pasado que han desaparecido, que están obsoletos o vaticinan otros que servirán de base para la fundación de nuevos modelos de sociedad** a partir del cambio paradigmático

* El sustrato metodológico que sustenta las afirmaciones del procedimiento sugerido para promover el cambio actitudinal aludido en el proceso transformativo hacia el Nuevo Humanismo es el contenido de una tesis doctoral que le significó a la autora acceder al grado de Doctora en Ciencias del Desarrollo Sustentable, titulada: "Modelo Teórico-Metodológico Transdisciplinario para sujetos complejos en tiempos de crisis (Método Implexo) para sujetos complejos en tiempos de crisis", Universidad de Guadalajara, México, febrero 2011.

* Categorías tales como era postmoderna (Etzioni), sociedad postburguesa (Lichtheim), sociedad posteconómica (Kahn), sociedad de la postescasez (Bookchin), sociedad postcivilizada (Boulding), sociedad postindustrial (Bell), sociedad del conocimiento (Drucker), sociedad de los servicios personales en curso. La crisis civilizatoria no es un secreto para nadie. La falta de equidad sostenida por el modelo de poder, las migraciones forzadas, la falta de inclusión, la crisis institucional y de los sistemas de representación política victimizan a millones de ciudadanos en el mundo y comprometen su sobrevivencia. Las deficiencias y deformidades de este patrón civilizatorio constituyen problemáticas globales comprensibles desde la mirada de la Complejidad. Y, aunque podríamos pensar que esta crisis es fundamentalmente responsabilidad de los poderosos, de las corporaciones económicas y oligopolios, de la clase política de turno, de los países del Norte, de las burocracias inerciales por su resistencia al Cambio... los Sujetos no estamos exentos de responsabilidades. Es una realidad evidente que la crisis del patrón civilizatorio de la que hablamos se alimenta de la dimensión personal que se expresa en el contexto de las interacciones sociales, y se ancla en el universo perceptivo y actitudinal de los sujetos. Autores como Capra o Pániker coinciden en que la crisis paradigmática es fundamentalmente una crisis de percepción. Por ello, es necesario reorientar las acciones de los sujetos a una nueva dirección comportamental para instalar un Nuevo Humanismo.

\section{Nuevo Humanismo, conciencia planetaria y sujetos complejos}

La necesidad de asumir y enfrentar dicha crisis ha llevado a Edgar Morin (2011) a sostener

(Halmos), sociedad clasista de servicios (Dahrendorf), era tecnotrónica (Brzezinski). (Mejía, 2003, p.190) 
a lo largo de su vasta obra que la solución es la promoción de un Nuevo Humanismo. Con este aparecería una Tercera Cultura que ha de reunir Artes y Ciencias* en un solo cuerpo cultural, puesto al servicio de la transformación de las conciencias, re-ligando de modo solidario y respetuoso los fenómenos complejos que han sido escindidos por la mirada simplificadora de nuestra civilización en crisis (Pozzoli, 2007, 2011). Este Nuevo Humanismo respeta la dignidad de todos los seres vivos en relación de hetarquía, reconoce su fragilidad, activa un sentimiento de fraternidad y solidaridad contenida en una conciencia planetaria que es en sí misma una propuesta ética de base para refundar una nueva civilización (Morin, 2011), presente en la subjetividad de los sujetos reales, en una forma de estar en el mundo más amable y solidaria que contribuya a la construcción de un mundo mejor (Pozzoli, 1999, 2006, 2007, 2011).

\section{Obstáculos, inercias y contradicciones en}

\section{los sujetos para la transformación}

Las intenciones del sujeto complejo de llevar al plano de su acción de un modo consistente la Teoría del Nuevo Humanismo, pueden quedar en el camino, ser bloqueadas o desalentadas al interior de los grupos de pertenencia a los que está integrado. El sistema tiene capacidad

\footnotetext{
Un claro ejemplo de ese Nuevo Humanismo, es la propuesta denominada Arte, belleza y espiritualidad desde el pensamiento complejo de María Teresa Pozzoli, obra que consta de 25 capítulos en los que Literatura, Cine, Ciencia, Cosmología, Neurobiología, Espiritualidad, se reúnen de un modo organizado en una trama reflexiva que promueve la transformación de las conciencias hacia un Nuevo Humanismo. Libro Publicado por Centro del Diálogo de Civilizaciones, Coquimbo, Chile, 2012.
}

de absorción de las conciencias, lo que hace que -poco o nada- cambie; de este modo las conciencias pueden quedar pasivas, frustradas y desencantadas e inhibir su transformación, predominando la convicción de que nada sirve, que no hay alternativa viable para esta crisis. $\mathrm{La}$ consecuencia de esta percepción es la tendencia a mantenerse en una respuesta acrítica, reproduciendo el modelo inercialmente. En palabras de Zemelman (2000):

En efecto, la inercia mental siempre ha frenado el cambio, la innovación y el progreso: la inercia mental se rinde y cede a la comodidad momentánea y a la ley del menor esfuerzo. (p.2)

Esta es una cita clave que nos permite introducirnos en la problemática del retraso del cambio y en el protagonismo que en ese retraso tienen, paradojalmente, los propios sujetos de la

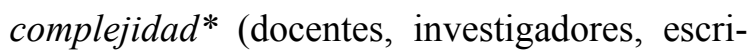
tores, difusores del Pensamiento Complejo). Esta paradoja tendría razón de ser en el cambio actitudinal implicado en esta transformación valórica hacia el Nuevo Humanismo **, dado que se trata de un fenómeno cruzado por múltiples factores psicosociales que, en esta ocasión, escapa al objetivo central de este trabajo.

* Cuando usamos la expresión sujeto complejo (Pozzoli, 2006) hacemos referencia al eje 'continuidad/ruptura con el pasado', pero también a la presencia o ausencia de aleatoriedad, de azar y de indeterminación, ante los que nuestros esquemas simplificadores de análisis colapsan.

** El cambio actitudinal es parte de lo que el Maestro Morin entiende al referirse a que "las cabezas estén bien puestas" (Morin, 1999). 
Que un Sujeto Complejo difunda discursivamente este pensamiento transformador y que, sin embargo, no sea coherente en su acción con las ideas de cambio que enuncia, muestra que permanece adaptado a la norma mayoritaria del paradigma en crisis que critica. Asumir la decisión de ajustar la propia vida a los valores de una teoría que pone en tela de juicio el pensamiento mayoritario, exige al sujeto complejo asumir el rol de transgresor para oponerse con su acción a la cultura dominante, llevando, así, al nivel de la acción, su mirada translógica*, lo que tendría claras consecuencias de sanción en grupos de pertenencia, organizaciones, etc.

En realidad, la mirada de la complejidad impone la re-organización del pensamiento y le demanda al sujeto constituirse, reorganizarse, como un sujeto cognoscente diferente al tipo medio educado en el paradigma simplificador**.

\section{El Pensamiento Complejo es una base} metateórica diferente para educar a las nuevas generaciones* en lo que respecta a una relación armónica con la Naturaleza, la concepción de las relaciones humanas y del Sí Mismo.

* Morin habla, no de la necesidad de superar la lógica clásica sino de "jugar un doble juego entre la lógica y su transgresión" (Morin, 1984, p.346).

** Para profundizar esa crítica, sugiero revisar El paradigma perdido 1999. Barcelona: Kairós.

* Desde la aplicación de esta Metodología (Método Implexo, Pozzoli, 2011) se han formado unas 4000 personas; educadores y profesionales de la salud, cuya acción ha tenido en sus grupos de pertenencia un efecto multiplicador orientado hacia la transformación paradigmática.
Necesidad de formación sociopersonal de

\section{los sujetos}

Toda explicación, mirada o enfoque teórico, contiene un modelo de ser humano. Tal modelo no está necesariamente explicitado; por el contrario, suele mantenerse en un nivel de borrosidad que se rescata al activar la innovación y la creatividad de quienes interpretan esos modelos teóricos. Ese modelo de ser humano ha de ser un recurso fundamental para quienes tienen entre sus objetivos de vida, la superación de sus limitaciones personales y la búsqueda del cambio social.

La transformación hacia un Nuevo Humanismo requiere la activación de una cultura de la formación sociopersonal, encarnada en la vida de los sujetos reales con quienes se convive. Este cambio actitudinal de los sujetos implica:

1. el desarrollo de su potencial y el mejoramiento integral de su calidad de vida

2. que el sujeto es el protagonista de su desarrollo, es un 'sujeto activo'

3. que el sujeto es un desarrollo integral que abarca las dimensiones bio-psico-socioespiritual

4. que el desarrollo personal no se refiere al ámbito curativo, sino que es promocional y preventivo.

En otros textos (Pozzoli, 1999, 2006) hemos desarrollado la idea de Educar sujetos complejos amables. Si los sujetos no asumen una sensi- 
bilidad más amigable y universal, sustentable, estaremos condenados inevitablemente a la regresión*, y a más de lo mismo. Justamente para ello es pertinente la noción de sustentabilidad $^{* *}$ que no es otra cosa que meollar para el pensamiento ecológico, y solo adquiere sentido al concebir la realidad como un ecosistema. El desarrollo de una conciencia planetaria del sujeto requiere de un re-aprendizaje, que es al mismo tiempo un des-aprendizaje, ampliamente descrito en el Método Implexo (Pozzoli, 2011) y del que presentamos algunos módulos que lo conforman:

* Para profundizar en estas ideas, sugiero la lectura: Pozzoli, M. T. (2007). El trato amable del sujeto complejo. Pensamiento Complejo y Conciencia Planetaria. Revista Académica de Psicología BABEL, 2, 182-205. ISSN 0718-0187.

** La noción de sustentabilidad permite introducir un referente para juzgar las instituciones y las prácticas vigentes en las sociedades modernas, poniendo en tela de juicio la calidad de la convivencia que emerge en cuanto existencia tóxica en el actual modelo de desarrollo. La noción de sustentabilidad es básica para el pensamiento ecológico, Pues los sistemas socioeconómicos han de ser reproducibles más allá del corto plazo, sin deterioro de los ecosistemas sobre los que se apoyan. Es decir, sustentabilidad es viabilidad ecológica: los sistemas socioeconómicos que funcionan destruyendo su base biofísica son insostenibles. asumir la incertidumbre, asimilar la contradicción, revisar los dogmas, practicar el ejercicio de la duda de sus certezas, optar por teorías abiertas y transitorias, comprender que todo ser vivo está en movimiento, y que lo que vive el sujeto es 'situacional', saber que su capacidad de auto-regenerarse es por ser una máquina viva, deconstruir su ideología, deconstruir el impacto del Funcionalismo en su Autoconcepto, reconocer creencias basadas en pensamiento clichés, estereotipos, prejuicios, mitos. Todas estas sujeciones tienen la bondad de que así como han sido aprendidas son transitorias y corregibles, porque mediante el Método Implexo pueden ser progresivamente des-aprendidas.

* "Construcción de un Modelo Teórico Metodológico Transdisciplinario (eco-psico-filo socio-histórico-educativo) de sujetos complejos en una sociedad hacia una conciencia planetaria" (Tesis Doctoral; para obtener el grado de Doctora en Ciencias del Desarrollo Sustentable). Centro Universitario de la Costa, Universidad de Guadalajara, México.

\section{a) Desaprender lo aprendido}

Implica que el Sujeto aprenda tomando ciertas decisiones respecto de su acción*.

\section{b) Reconocer inhibiciones y bloqueos de la} subjetividad

La razón principal que motivó el Método Implexo fue una pregunta moriniana que asumí como propia: ¿Quién educará a los educadores?

Al respecto, cabe precisar que la verdadera transformación paradigmática no puede ser por decreto, debe ocurrir por la propia decisión de cambio de los sujetos, en la cabeza de la gente. Sin embargo, en este proceso, el sujeto complejo ha de enfrentar ineludiblemente una etapa evitativa.

Lo anterior se debe a que toda desconfirmación de una parte de lo que somos, de lo que consideramos verdadero durante una parte fundamental de nuestra vida,...el descubrimiento de porqué pienso lo que pienso (Cibernética de 2 do. orden), nos permite develar nuestros prejuicios, es insegurizante y moviliza mecanismos defensivos.

\footnotetext{
Acciones relacionadas con: asumir la incertidumbre, asimilar la contradicción, revisar los dogmas, practicar el ejercicio de la duda de sus certezas, optar por teorías abiertas y transitorias, comprender que todo ser vivo está en movimiento, y que lo que vive el sujeto es 'situacional', saber que su capacidad de auto-regenerarse es por ser una máquina viva, deconstruir su ideología, deconstruir el impacto del Funcionalismo en su Autoconcepto, reconocer creencias basadas en pensamiento clichés, estereotipos, prejuicios, mitos. Todas estas sujeciones tienen la bondad de que así como han sido aprendidas son transitorias y corregibles, porque mediante el Método Implexo pueden ser progresivamente des-aprendidas.
} 
El Método Implexo reúne un conjunto de herramientas reflexivas que facilitan el descubrimiento de esas inhibiciones, pero también propone caminos para su remoción, de corregir la ceguera de los supuestos del paradigma*, cuando el Sujeto tome la decisión respectiva.

Quienes creemos representar el Pensamiento Complejo, los 'progresistas'... los pioneros del cambio... -dada la capacidad externalizante del paradigma aprendido- solemos ignorar espontáneamente la propia sujeción, el dispositivo de control funcionalista montado en la conciencia y en la acción de la vida cotidiana.

En este sentido, la disonancia cognoscitiva** (Festinger, 1957) forma parte del proceso de transformación actitudinal; impone una tensión, por la contradicción existente entre lo que afirmamos discursivamente y la orientación real que le damos a la acción adaptada al Sistema.

c) Niveles de desarrollo moral superior de sujetos amables

Alcanzar el Nuevo Humanismo implicará aumentar los niveles de desarrollo moral. Ello significa que el sujeto complejo habrá de elevar su desarrollo moral al nivel postconvencional

* Esto nos recuerda la afirmación de Cecilia Dockendorf; “... el paradigma es como el agua que el pez no puede ver porque siempre ha estado dentro de ella" (Dockendorf, 1988).

** El concepto de disonancia cognitiva hace referencia a la tensión o desarmonía interna del sistema de ideas, creencias y emociones (cogniciones) que percibe una persona al concebir al mismo tiempo dos pensamientos que están en conflicto, o por un comportamiento que entra en conflicto con sus creencias. Es decir, el término se refiere a la percepción de incompatibilidad de dos cogniciones simultáneas, que impacta en sus actitudes y en sus acciones.
(Kölhberg, 1992). Esto constituye un universo actitudinal con presencia de principios de tipo universal respecto de la Ecología, la Justicia, la Libertad, la Solidaridad, el respeto inalienable a los Derechos de lo Vivo.

\section{d) Ejercicio metacognitivo}

El sujeto reconoce su condición de complejo mediante el ejercicio metacognitivo, es decir, a través de su multidimensionalidad interior, ampliando su universo actitudinal en razón del reconocimiento de su Complejidad*. Este ejercicio tiene consecuencias en su Autoconcepto, en su biografia, en sus expectativas existenciales, en la aceptación del Otro (diferente), en la receptividad y defensa de los derechos de los seres vivos.

e) Ruptura y liberación **

La práctica de autoobservación, de trabajar con la propia identidad, implica realizar una cirugía mayor respecto a la propia interioridad.

El sujeto integra nuevas bases matriciales al integrar conocimientos viejos y nuevos en una nueva organización del pensamiento. Para ello, es necesaria la ruptura, la autocrítica y reconstrucción en tres dimensiones: con la verdad

* En el artículo "El trato amable del sujeto complejo", Revista Polis, Santiago, Chile, 2007, se describe una Matriz Actitudinal, a saber: acoger, recibir, respetar, bien-estar, reflexionar, ser parte, integrar, asumir responsabilidad, etc. En contraposición a la matriz actitudinal del 'guerrero' que es el sujeto protagonista del actual patrón civilizatorio en crisis.

** Para Freire (1970), la reflexión acerca de la propia realidad a lo largo del proceso educativo da cabida a la liberación, que se realiza en el re-encuentro de la persona con su propia dignidad como ser cultural, creador y transformador de la misma y en la construcción de la conciencia de sí misma. 
absoluta, con la mirada única, con la racionalidad técnica como única forma de conocimiento.

\section{f) Reflexión autobiográfica}

Se trata de un ejercicio de autoobservación, de autobiografía, de descubrimiento de la propia experiencia de forma narrativa, que significa reconocer un sentido a la trayectoria personal y de proyecto de vida.

\section{g) Ser mejor persona y estar más integrado}

Hacer realidad el Nuevo Humanismo implica que el Sujeto Complejo decida trabajar por su superación personal. Ser mejor persona va de la mano de estar más integrado por lograr mayores niveles de congruencia* entre los niveles discursivo/enunciativos y nuestras acciones cotidianas. El hecho de posicionar su juicio crítico en un estadio superior de desarrollo moral, muchas veces lleva a remar contra la corriente. Según Kölhberg, en el Nivel Postconvencional, la persona representa los principios en los que se basa cualquier sociedad buena, con mayores niveles de autonomía personal respecto de la presión grupal.

h) El sujeto ejerce un impacto modelar sistémico

Las acciones de quienes difunden $\mathrm{y}$ promueven el Pensamiento Complejo tienen un

* La congruencia es la armonía y el balance que existe entre nuestras acciones, pensamientos y emociones; las acciones son un reflejo de nuestros pensamientos y emociones, como emergente de una toma de conciencia de los procesos por los cuales los sujetos transitan (Pozzoli, M. A. (2003). Apuntes de Cátedra de Psicología Social. Escuela de Psicología, Universidad Academia de Humanismo Cristiano, Santiago, Chile) impacto 'modelar' para la vida de los sujetos con quienes interactúan o, con otras palabras, un impacto sistémico en términos culturales.

\section{Conclusión}

Vivimos tiempos que no admiten más renuncias ni neutralidades. Las teorías deben sustanciarse en los procesos de vida para que tengan algún sentido que supere lo meramente retórico. Son tiempos de pensar en vías alternas y creativas, que nos permitan desarrollar competencias y capacidades que rompan con los límites establecidos por este patrón civilizatorio, simplificador, opresor y reductivo (Ghiso, 2002).

Es imperativo plantear y respondernos la pregunta sobre cómo promover y mantener en las personas una disposición a pensar y pensarse, a observar y observarse, a construir y construirse en condiciones y dinámicas socioculturales que nos permitan sobrevivir con dignidad a nuestra propia precariedad.

No podemos olvidar que el paradigma simplificador a superar es externalizante, lo que implica el explícito descuido de la interioridad, la inhibición de la autoobservación y la delegación atribucional -en otros- de las propias responsabilidades a modo de rictus impersonal y externo.

En las experiencias educativas, poco o nada se alientan las prácticas reflexivas, de la autoobservación, y menos aún la utilización de los recursos emocionales e intuitivos como fuente de enriquecimiento experiencial (Pozzoli, 2007). 
Se trata de estimular una nueva manera de pensar, que no se limite a separar para conocer y en su lugar vincule, que ligue lo que está separado, como por ejemplo, las teorías que enunciamos y difundimos con las acciones de la propia vida. Hacer esta ligazón le permitirá a los sujetos del Nuevo Humanismo, evidenciar que no se trata de mera charlatanería, al mismo tiempo de ser más autónomos respecto de los supuestos del paradigma que nos sojuzgan la conciencia y la acción.

Las disciplinas académicas suelen magnificar el papel del conocimiento tecno-científico y mirar despectivamente el sentido común, prejuiciando -o ignorando prácticamente- el campo de la interioridad, al considerarlo un aspecto relacionado con lo mítico, lo esotérico o lo religioso (Mora, 2007).

La labor pendiente es, por ello, la construcción de sí mismo como fenómeno de superación individual, que en el Unitas Multiplex es también una exigencia colectiva, y en ella radica la importancia de otro aspecto fundamental del mensaje del Nuevo Humanismo: la ineludible dimensión colectiva que conlleva toda vida humana, por ser parte de la trama de complejidad, por ser una vida entramada y una conciencia planetaria llevada a la acción.

La pregunta clave que nos queda como corolario es: ¿en qué acciones de nuestra vida cotidiana se evidencia que hemos adoptado el Pensamiento Complejo para mirar el mundo?
Estamos ante una crisis que exige una revolución ética que nos traspasa la vida personal, fundada en compromisos efectivos que necesariamente requerirán de decisiones personales y existenciales, consistentes y congruentes con las ideas que difundimos: nuevos saberes, nuevos métodos, nuevos sentidos de la profesión de educar, pero sobre todo, una actitud activa para crear nuevos espacios de justicia y solidaridad en nuestros grupos de pertenencia. Ya no es posible separar la vida personal de la vida profesional, del rol de ciudadano, somos seres multidimensionales, pero al mismo tiempo integrados.

Los tiempos que corren, posibilitarán implementación de herramientas metodológicas en los espacios educativos del tipo de las del Método Implexo, y estos han de colaborar con esta transformación, que es la razón de haberlo modelizado y aplicado a escala. Corresponde pues a la educación formal e informal en todos sus espacios institucionales y sociales, desde la familia a la escuela, las interacciones cotidianas, en la universidad y en el mundo laboral, activar todos los procesos y acondicionar todos los ambientes capaces de estimular y hacer posible una regeneración multidimensional de nuestra conciencia planetaria de sujetos complejos que abarque e integre al mismo tiempo lo personal y lo social, junto a lo político, lo económico y lo ecológico.

\section{Referencias}

Boff, L. (2001). Ética planetaria desde el Gran Sur. Madrid: Editorial Trotta. 
Dockendorf, C. (1988). Notas sobre la noción de paradigma, en La fuerza del Arco Iris. Santiago de Chile: Jorge Osorio y Luis Weinstein Editores, (nstitute of Social Studies de la Haya (ISS) y Consejo de Educación de Adultos de América Latina (CEAAL).

Festinger, L. (1957). A theory of cognitive dissonance. Stanford, CA: Stanford University Press. ISBN 978-0-8047-0911-8.

Freire, P. (1970): La educación de los adultos como acción cultural. Introducción a su comprensión. Estados unidos: Universidad de Harvard.

Ghiso, A. (2002). Para enfrentar la indiferencia... una configuración epistémica humana y compleja. En línea, Medellín: FUNLAM FIUC. Disponible en: www. quadernsdigitals.net.

Kohlberg, L. (1992). Psicología del desarrollo moral. Bilbao: Desclée de Brouwer.

Leff, E. (2007). Aventuras de la Epistemología Ambiental. México: Siglo XXI Editores.

Luján, J \& Echeverría, J. (2004). Gobernar los riesgos. Ciencia y valores en la sociedad del riesgo. España: OEI-Biblioteca Nueva.

Mora, W.M. (2007). Respuesta de la universidad a los problemas socioambientales: la ambientalización del currículo en la educación superior. Revista Investigación en la Escuela. (Sevilla-España). 63,(3), 65-76.

Morin, E. (1999). Los siete saberes necesarios para la educación del futuro. Francia: Organización de las Naciones Unidas para la Educación, la Ciencia y la Cultura.
Morin, E. (2011). La vía para el futuro de la humanidad. Barcelona: Editorial Paidós.

Pozzoli, M. (1999). Complexus: Psicología, ciencias de la salud y cambio cultural: desde el paradigma de la complejidad. Santiago de Chile: LOM.

Pozzoli, M.T. (2000). La interacción social, una nueva epistemología para el cambio social. Castalia. Revista de Psicología de la Academia 1(1), 47-58.

Pozzoli, M. A. (2003). Apuntes de Cátedra de Psicología Social. Escuela de Psicología, Santiago, Chile: Universidad Academia de Humanismo Cristiano.

Pozzoli, M.T. (2006). El sujeto de la complejidad. La construcción de un Modelo Teórico Transdisciplinar (eco-psicosocio-histórico-educativo). Revista Polis, 5(15), 205-225.

Pozzoli, M.T. (2007). Transformar el conocimiento en la sociedad globalizada: Pensamiento complejo y transdisciplinariedad Revista Polis 6,(16), 163-196. ISSN 0717-6554

Pozzoli, M.T. (2007). El trato amable del sujeto complejo. Pensamiento complejo y Conciencia Planetaria. Revista Académica “BABEL”, 2, 182-205. ISSN 0718-0187.

Pozzoli, M.T. (2008). El Pensamiento Complejo y la emergencia de una eco-ética para un mundo de la vida 'buena', en Sociedad y mundo de la vida a la luz del Pensamiento Fenomenológico-hermenéutico actual. Santiago: Ediciones Universidad Cardenal Silva Henríquez. 
Pozzoli, M.T. (2011). El aprendizaje del Pensamiento Complejo: como vía de acceso a la salud integral. Libro colectivo Complejidad y América Latina. Santiago de Chile: Comunidad Internacional de Pensamiento Complejo, 2011.

Pozzoli, M.T. (2007). Espiritualidad, arte y belleza. Espacios del universo para el desarrollo humano desde el pensamiento complejo Revista de la Universidad Bolivariana, 7(17), 117-136. ISSN 0717-6554.

Pozzoli, M.T. (2011)

Modelo Teórico-Metodológico Transdisciplinario. (Método Implexo) para sujetos complejos en tiempos de crisis. Revista Sustentabilidad(es), 6.
Pozzoli, M. (2011). "Construcción de un Modelo Teórico Metodológico Transdicciplinario (eco-psico-filo socio-histórico-educativo) de sujetos complejos en una sociedad hacia una conciencia planetaria"; Tesis Doctoral para obtener el grado de Doctora en Ciencias del Desarrollo Sustentable. Centro Universitario de la Costa, Universidad de Guadalajara, México.

Pozzoli, M.T. (2012). Arte, belleza y espiritualidad desde el pensamiento complejo. Coquimbo, Chile: Centro del Diálogo de Civilizaciones.

Riechmann, J. (2006). Dimensiones profundas de la sostenibilidad. Rincones del Atlántico, 3, 234-235.

Zemelman, H. (2000). Debate sobre la situación actual de las Ciencias Sociales. Análisis y reflexiones año $I I I,(003)$, julio-septiembre. 